\title{
新的 $2^{9}$ 阶旗传递仿射平面
}

献给冯克勤教授 80 华诞

\section{冯涛 ${ }^{1}$, 李伟聪 ${ }^{2}$, 向青 $2 *$}

1. 浙江大学数学科学学院, 杭州 310027 ;

2. 南方科技大学数学系, 深圳 518055

E-mail: tfeng@zju.edu.cn, liwc3@sustech.edu.cn, xiangq@sustech.edu.cn

收稿日期: 2021-02-22；接受日期: 2021-06-09；网络出版日期: 2021-09-22；＊通信作者 国家自然科学基金 (批准号: 11771392, 12071296 和 M-0157) 资助项目

摘要 有限旗传递仿射平面与很多组合对象 (展形、平面函数、半域和线性化多项式等) 有着密切联 系, 因而在过去 50 多年来受到研究者的广泛关注. Foulser 在 1964 年已经完整地确定了有限旗传递仿 射平面的自同构群. 如果一个旗传递仿射平面有一个可解自同构群, 则称该平面是可解的, 否则称它 是不可解的. 不可解的情形早在 20 世纪 90 年代末已经给出了完整的分类, 而可解的情形至今难以给 出完整的分类. 目前所有已知的可解旗传递仿射平面可以分为两类: $\mathcal{C}$-平面和 $\mathcal{H}$-平面, 其中 $\mathcal{H}$-平面 只在奇特征的情形下出现. 本文的主要贡献是首次构造出 $2^{9}$ 阶的非 $\mathcal{C}$ 型旗传递仿射平面并确定了其 全自同构群.

关键词 仿射平面 旗传递 展形 (spread) 平移平面

MSC (2020) 主题分类 $51 \mathrm{E} 15,05 \mathrm{~B} 25,51 \mathrm{~A} 40$

\section{1 引言}

一个 $m$ 阶仿射平面是一个点与线的关联结构 $(\mathcal{P}, \mathcal{L})$, 满足下述条件: (1) 任意两个点落在唯一的 一条线上; (2) 过每个点有 $m+1$ 条线, 每条线包含 $m$ 个点; (3) 对于不关联的点线对 $(P, \ell)$, 存在唯一 的过 $P$ 的线与 $\ell$ 不相交. 它也可以看作是一个参数为 $2-\left(m^{2}, m, 1\right)$ 的 2 - 设计. 本文考虑一类特殊的 仿射平面, 即平移平面 (translation planes). 平移平面在过去的 50 年中已被广泛研究, 参见文献 $[1,2]$.

平移平面可以用群论的语言来描述, 这是 André 在文献 [3] 中首次给出的. 令 $A$ 是一个 $m^{2}$ 阶 Abel 加法群. 令 $\mathcal{S}=\left\{W_{0}, W_{1}, \ldots, W_{m}\right\}$, 其中每个 $W_{i}$ 是 $A$ 的 $m$ 阶子群 (即 $\left|W_{i}\right|=m$ ), 满足下面的两个条 件: (1) 对于任意 $0 \leqslant i<j \leqslant m$ 有 $W_{i} \cap W_{j}=\{0\} ;(2) A \backslash\{0\}=\bigcup_{i=0}^{m} W_{i} \backslash\{0\}$. 这样的集合 $\mathcal{S}$ 被称为群 $A$ 的一个展形 (spread). 这些子群 $W_{i}$ 也被称为展形 $\mathcal{S}$ 的组件 (component). André 利用展形 $\mathcal{S}$ 构造出以 $A$ 为点集、 $\{v+W: v \in A, W \in \mathcal{S}\}$ 为线集的关联结构, 其中关联关系是包含关系. 这样的结构即为一 
个以 $A$ 为平移群的平移平面 $\Pi(\mathcal{S})$, 可以证明具有上述展形 $\mathcal{S}$ 的 $A$ 必为初等 Abel 群, 而且 $\Pi(\mathcal{S})$ 的阶 必为素数幂. 该平面的核或者说展形的核定义为 $\operatorname{ker}(\mathcal{S}):=\{g \in \operatorname{End}(A): g(W) \subseteq W, \forall W \in \mathcal{S}\}$. 可以证 明对于有限阶的 $\Pi(\mathcal{S}), \operatorname{ker}(\mathcal{S})$ 是一个有限域. 这种构造平移平面的方法在文献中称为 André 构造法.

令 $q=p^{f}$ 为素数幂, $\mathbb{F}_{q}$ 是含有 $q$ 个元素的有限域, 其中 $p$ 是素数. 令 $V$ 是在 $\mathbb{F}_{q}$ 上的 $2 n$ 维的 线性空间, 它也可以看成是一个 Abel 加法群. 定义 $\Gamma \mathrm{L}(V)$ 为 $V$ 上所有可逆的 $\mathbb{F}_{q^{-}}$半线性变换. 这里 $V$ 的一个展形 $\mathcal{S}$ 即为划分 $V$ 中所有非零向量的 $n$ 维子空间 $W_{i}$ 构成的集合. 由于域的特征是有限 的, $\operatorname{ker}(\mathcal{S})$ 是包含 $\mathbb{F}_{q}$ 的有限域. 全自同构群 $\operatorname{Aut}(\mathcal{S})$ 是 $\Gamma \mathrm{L}(V)$ 中所有把组件映到组件的线性变换构 成的子群. 由 André 构造法得到的仿射平面是一个 $q^{n}$ 阶平移平面 $\Pi(\mathcal{S})$. 全自同构群 $\operatorname{Aut}(\mathcal{S})$ 也被称 为该平面的平移补 (translation complement). 令 $\mathcal{S}$ 和 $\mathcal{S}^{\prime}$ 是 $V$ 的两个展形. 如果存在 $g \in \Gamma \mathrm{L}(V)$ 使得 $\mathcal{S}^{\prime}=\{g(W): W \in \mathcal{S}\}$, 那么这两个展形是同构的. 由同构的展形所生成的仿射平面也是同构的.

仿射平面的旗 (flag) 是满足 $P \in \ell$ 的点线对 $(P, \ell)$. 如果一个仿射平面的直射群 (collineation group) 在这些旗上的作用是传递的, 那么该平面被称为旗传递的. 本文考虑的平面都是有限的. 1965 年 Wagner ${ }^{[4]}$ 证明了所有旗传递仿射平面必须是平移平面, 同时它们必然是由 $\mathbb{F}_{q}$ 上 $2 n$ 维线性空间 $V$ 中的展形 $\mathcal{S}$ 所得到的, 并且它们的阶都是素数幂. 由展形 $\mathcal{S}$ 所构造出来的仿射平面 $\Pi(\mathcal{S})$ 是旗传 递的当且仅当 $\operatorname{Aut}(\mathcal{S})$ 在 $\mathcal{S}$ 上的作用是传递的. 具有不可解自同构群的旗传递仿射平面早在 20 世纪 90 年代就已经被完全分类了: 它们分别是 Desarguesian 平面、Lüneburg 平面、9 阶拟域平面和 27 阶 Hering 平面 (参见文献 $[5,6]$ ). Foulser [7,8] 已经确定了有限旗传递仿射平面的自同构群. 同时他也证 明了可解旗传递仿射平面的平移补同构于一维仿射半线性群 $\operatorname{A\Gamma L}\left(1, q^{2 n}\right)$ 的子群, 其中 $q$ 是素数幂.

可解旗传递仿射平面的完整的分类一直是有限几何领域中的公开问题. Kantor 和 Suetake 在一系 列文献 [9-12] 中给出了很多非经典 (non-Desarguesian) 奇数阶的旗传递仿射平面的构造, 这类平面在 文献 [13] 中被称为 Kantor-Suetake 平面. Kantor 和 Williams ${ }^{[14]}$ 利用辛展形 (symplectic spreads) 构 造出大量偶数阶的旗传递仿射平面, 但是这些构造所产生的仿射平面在核上的维数都是奇数. 对于偶 数维旗传递仿射平面, 只有维数为 2 的情形被 Baker 和 Ebert ${ }^{[15,16]}$ 完整地分类. 当域的特征是偶数 时, 是否存在其他偶数维非经典旗传递仿射平面仍然是一个公开问题. 特别地, 目前已知的可解旗传 递仿射平面对应的展形 $\mathcal{S}$ 的全自同构群 $\operatorname{Aut}(\mathcal{S})$ 包含一个 “大的” Singer 子群, 其中这个子群要么在 $\mathcal{S}$ 上的作用是传递的, 要么在 $\mathcal{S}$ 上恰好有两个长度一样的轨道. 这两类仿射平面在文献中分别被称为 $\mathcal{C}$ - 平面和 $\mathcal{H}$ - 平面. 如果仿射平面的阶和维数满足特定的公因子条件, 这样的旗传递仿射平面只可能 是 $\mathcal{C}$ - 平面或 $\mathcal{H}$ - 平面. 这两类平面在过去的 20 年内被广泛地研究. 特别地, 如果旗传递仿射平面是 奇数阶的并且在核上的维数是 3 维, 文献 [17-20] 利用一些几何方法说明这种情形只会出现 $\mathcal{C}$ - 平面和 $\mathcal{H}$ - 平面. Prince 曾尝试去寻找新的可解旗传递仿射平面, 他在文献 [21] 中确定了所有 $n \leqslant 125$ 阶旗 传递仿射平面, 并说明了这些平面都是已知的. 最近, Feng ${ }^{[13]}$ 结合平面函数以及置换多项式的研究提 出了一种新方法去刻画和研究旗传递仿射平面, 这种方法提供了解决偶特征下偶数阶 $\mathcal{C}$ - 平面的分类 的想法.

本文考虑可解旗传递仿射平面. 第 2 节介绍一些基本概念以及简要地回顾已知的构造. 第 3 节给 出新的 $2^{9}$ 阶旗传递仿射平面的构造, 并确定它的全自同构群. 最后对全文进行总结.

\section{2 准备工作和已知的构造}

令 $V=\mathbb{F}_{q^{2 n}}$ 是 $\mathbb{F}_{q}$ 上的 $2 n$ 维线性空间, 再令 $\gamma$ 是 $\mathbb{F}_{q^{2 n}}$ 的一个本原元, 其中 $q=p^{f}, p$ 是一个素 
数; $n \geqslant 2$ 是正整数. 定义 $V$ 上的两个线性变换:

$$
\rho_{a}: x \mapsto a x \quad \text { 和 } \quad \sigma: x \mapsto x^{p},
$$

其中 $a \in \mathbb{F}_{q^{2 n}}^{*}, \rho_{a}$ 和 $\sigma$ 都是从左边作用的线性变换. 因此有 $\sigma \rho_{a} \sigma^{-1}=\rho_{a}^{p}=\rho_{a^{p}}$. 这里为了简洁记 $\rho=\rho_{\gamma}$, 即 $\rho(x)=\gamma x$. 假定 $\Pi(\mathcal{S})$ 是由 $V$ 中的展形 $\mathcal{S}$ 所得到的仿射平面. 根据 Foulser ${ }^{[7]}$ 的结果可知, 如果 $\Pi(\mathcal{S})$ 是一个具有可解自同构群的旗传递仿射平面, 那么 $\operatorname{Aut}(\Pi(\mathcal{S}))$ 同构于 $A \Gamma \mathrm{L}\left(1, q^{2 n}\right)$ 的某一 个子群. 换言之, $\operatorname{Aut}(\mathcal{S})$ 同构于 $\Gamma \mathrm{L}\left(1, q^{2 n}\right)$ 的某一个子群. 因为 $\langle\rho, \sigma\rangle$ 与 $\Gamma \mathrm{L}\left(1, q^{2 n}\right)$ 是同构的, 所以 在同构等价意义下可以选取一个合适的展形 $\mathcal{S}$, 使得 $\operatorname{Aut}(\mathcal{S})$ 是 $\langle\rho, \sigma\rangle$ 的子群. Prince ${ }^{[21]}$ 已经确定了 $\operatorname{Aut}(\mathcal{S})$ 的具体形式, 但由于文献 $[21]$ 中有一些笔误, 这里再次给出证明.

引理 $2.1^{[21]}$ 令 $\mathcal{S}$ 是 $2 n$ 维 $\mathbb{F}_{q^{-}}$线性空间 $\mathbb{F}_{q^{2 n}}$ 使得 $\operatorname{ker}(\mathcal{S})=\mathbb{F}_{q}$ 的展形, 其中 $q=p^{f}$. 假设 $\Pi(\mathcal{S})$ 是由 $\mathcal{S}$ 定义的 $q^{n}$ 阶旗传递仿射平面. 如果 $\Pi(\mathcal{S})$ 的自同构群是可解的, 那么 $\mathcal{S}$ 的全自同构群 $\operatorname{Aut}(\mathcal{S})$ 同构于 $\left\langle\rho^{d\left(q^{n}-1\right) /(q-1)}, \rho^{a} \sigma^{s}\right\rangle$, 其中 $s$ 整除 $2 n f, d$ 整除 $\operatorname{gcd}\left(q^{n}+1,2 n f\right), 0 \leqslant a<d \frac{q^{n}-1}{q-1}$.

证明 不妨假设 $\operatorname{Aut}(\mathcal{S})$ 是 $\langle\rho, \sigma\rangle$ 的子群. 令 $k$ 是使得 $\operatorname{Aut}(\mathcal{S}) \cap\langle\rho\rangle=\left\langle\rho^{k}\right\rangle$ 的最小的正整数. 假定 $\operatorname{Aut}(\mathcal{S})$ 的阶是 $m \frac{q^{2 n}-1}{k}$. 考虑群同态 $\psi: \Gamma L\left(1, q^{2 n}\right) \rightarrow \operatorname{Gal}\left(\mathbb{F}_{q^{2 n}} / \mathbb{F}_{p}\right)$, 此处 $\psi\left(\rho^{i} \sigma^{j}\right)=\sigma^{j}$. 那么 $\psi(\operatorname{Aut}(\mathcal{S}))$ 是 $\operatorname{Gal}\left(\mathbb{F}_{q^{2 n}} / \mathbb{F}_{p}\right)$ 的 $m$ 阶子群. 因此存在 $s=2 n f / m$ 和 $0 \leqslant a<k$ 使得 $\operatorname{Aut}(\mathcal{S})=\left\langle\rho^{k}, \rho^{a} \sigma^{s}\right\rangle$.

因为 $\operatorname{ker}(\mathcal{S})=\mathbb{F}_{q}$, 所以 $\mathcal{S}$ 的每个组件是 $\mathbb{F}_{q^{-}}$线性的, 但不可能在 $\mathbb{F}_{q^{2 n}}$ 中更大的子域上线性. 于 是由 $\operatorname{ker}(\mathcal{S})$ 的定义可知 $\mathcal{S}$ 的每个组件在 $\langle\rho\rangle$ 中的稳定子群是 $\left\langle\rho^{\left(q^{2 n}-1\right) /(q-1)}\right\rangle$. 因此每个组件在 $\left\langle\rho^{k}\right\rangle$ 作用下的轨道大小都是 $\frac{q^{2 n}-1}{k(q-1)}$, 并且每个轨道是部分展形. 令 $d$ 是这些轨道的个数. 记 $|\mathcal{S}|=q^{n}+1$, 那么存在 $d$ 个大小为 $\frac{q^{n}+1}{d}$ 的 $\left\langle\rho^{k}\right\rangle$ - 轨道使得它们的并恰好是整个展形. 由于 $\operatorname{Aut}(\mathcal{S})$ 在 $\mathcal{S}$ 上的作用 是传递的, 因此 $\operatorname{Aut}(\mathcal{S}) /\left\langle\rho^{k}\right\rangle$ 在这些部分展形上的作用是传递的. 故 $d s \mid 2 n f$. 根据 $\frac{q^{n}+1}{d}=\frac{q^{2 n}-1}{k(q-1)}$, 我 们推导出 $k=d\left(q^{n}-1\right) /(q-1)$ 以及 $d$ 是 $\operatorname{gcd}\left(q^{n}+1,2 n f\right)$ 的一个因子. 证毕.

如果参数 $p 、 f$ 和 $n$ 满足

$$
\operatorname{gcd}\left(q^{n}+1,2 n f\right) \in\{1,2\}
$$

那么引理 2.1 中的参数 $d$ 只有两种可能: $d=1$ 或 $d=2$, 即 $\operatorname{Aut}(\mathcal{S})$ 中一个最大的 Singer 子群是 $\left\langle\rho^{\left(q^{n}-1\right) /(q-1)}\right\rangle$ 或 $\left\langle\rho^{2\left(q^{n}-1\right) /(q-1)}\right\rangle$. 于是根据文献 [20, 引理 1] 和后面的讨论 (或文献 [13, 引理 3.1]), 我 们推导出在这种情形下以 $\left\langle\rho^{d\left(q^{n}-1\right) /(q-1)}, \rho^{a} \sigma^{s}\right\rangle$ 为自同构群的旗传递仿射平面只有两种可能: $\mathcal{C}$ - 平面 或 $\mathcal{H}$ - 平面. 在接下来的讨论中, 我们列出所有已知构造中展形 $\mathcal{S}$ 的一个组件 $W$ 以及 $\operatorname{Aut}(\mathcal{S})$ 的一个 传递子群 $H$.

\subsection{Kantor-Suetake 构造}

下面继续使用上面定义的符号, 并令 $\sigma_{1}$ 是 Galois 群 $\operatorname{Gal}\left(\mathbb{F}_{q^{2 n}} / \mathbb{F}_{q}\right)$ 的一个元素, 即 $\sigma_{1}=\sigma^{f i}$, 其中 $0 \leqslant i<2 n$.

(1) 假设 $q$ 和 $n$ 都是奇数. 选取 $\mathbb{F}_{q^{2 n}}$ 的一个元素 $\delta$ 使得 $\delta+\delta^{q^{n}}=0$. 令 $W=\left\{x+\delta \sigma_{1}(x): x \in \mathbb{F}_{q^{n}}\right\}$ 和 $H=\left\langle\rho^{\left(q^{n}-1\right) /(q-1)}\right\rangle$. 那么 $\{g(W): g \in H\}$ 构成一个旗传递仿射平面的展形.

(2) 假设 $q$ 是偶数和 $n$ 是奇数. 选取 $\mathbb{F}_{q^{2}} \backslash \mathbb{F}_{q}$ 中的一个元素 $r$, 并令 $\operatorname{Tr}_{\mathbb{F}_{q^{n}} / \mathbb{F}_{q}}$ 是从 $\mathbb{F}_{q^{n}}$ 到 $\mathbb{F}_{q}$ 的迹 映射, 即 $\operatorname{Tr}_{\mathbb{F}_{q^{n}} / \mathbb{F}_{q}}(x)=\sum_{i=0}^{n-1} x^{q^{i}}, x \in \mathbb{F}_{q^{n}}$. 令 $W=\left\{\operatorname{Tr}_{\mathbb{F}_{q^{n}} / \mathbb{F}_{q}}(x)+r x: x \in \mathbb{F}_{q^{n}}\right\}$ 和 $H=\left\langle\rho^{\left(q^{n}-1\right) /(q-1)}\right\rangle$. 那么 $\{g(W): g \in H\}$ 构成一个旗传递仿射平面的展形. 
(3) 假设 $q^{n} \equiv 1(\bmod 4)$, 并选取 $\mathbb{F}_{q^{2 n}}$ 中的一个元素 $\delta$ 使得 $\delta+\delta^{q^{n}}=0$. 令 $W=\left\{x+\delta \sigma_{1}(x): x\right.$ $\left.\in \mathbb{F}_{q^{n}}\right\}$ 和 $H=\left\langle\rho^{2\left(q^{n}-1\right) /(q-1)}, \rho_{\delta} \sigma_{1}\right\rangle$, 其中 $\rho_{\delta}$ 如 $(2.1)$ 所示. 那么 $\{g(W): g \in H\}$ 构成一个旗传递仿 射平面的展形.

(4) 假设 $q^{n} \equiv 3(\bmod 4)$, 并选取 $\mathbb{F}_{q^{2 n}}$ 中的一个元素 $\delta$ 使得 $\delta+\delta^{q^{n}}=0$. 再取 $u \in \mathbb{F}_{q^{2 n}}$ 使得 $\sigma_{1}\left(u^{q^{n}+1}\right)=u^{q^{n}+1}$ 和 $u$ 不是 $\mathbb{F}_{q^{2 n}}$ 中的平方元. 令 $W=\left\{x+\delta \sigma_{1}(x): x \in \mathbb{F}_{q^{n}}\right\}$ 和 $H=\left\langle\rho^{2\left(q^{n}-1\right) /(q-1)}\right.$, $\left.\rho_{u} \sigma^{f n}\right\rangle$, 其中 $\rho_{u}(x)=u x$ 和 $\sigma^{f n}(x)=x^{q^{n}}$. 那么 $\{g(W): g \in H\}$ 构成一个旗传递仿射平面的展形.

在 Kantor-Suetake 类平面中, 构造 (1) 和 (2) 得到的是 $\mathcal{C}$ - 平面, 而构造 (3) 和 (4) 得到的是 $\mathcal{H}$ 平面.

\subsection{Kantor-Williams 构造}

假定 $q=2^{f}, n$ 是奇数和 $f$ 是正整数, 令 $\mathbb{F}_{q^{n}}=F_{0} \supset F_{1} \supset \cdots \supset F_{m}$ 是包含 $m+1$ 个有限域的 有限域链, 并且对每个整数 $0 \leqslant i \leqslant m$ 都有扩张次数 $\left[F_{0}: F_{i}\right]$ 是奇数. 再定义 $\operatorname{Tr}_{i}$ 是从 $F_{0}$ 到 $F_{i}$ 的 迹函数, 记 $K_{i}:=\left.\operatorname{ker} \operatorname{Tr}_{i+1}\right|_{F_{i}}$. 对每个整数 $0 \leqslant i \leqslant m$, 记 $F_{i}^{(2)}$ 是 $F_{i}$ 的二次扩域. 因为 $\left[F_{0}: F_{i}\right]$ 是奇 数, 所以 $F_{i}^{(2)}$ 是 $F_{0}^{(2)}$ 的子域. 定义 $C:=\left\{x \in \mathbb{F}_{q^{2 n}}: x^{q^{n}+1}=1\right\}$, 这恰好是 $\mathbb{F}_{q^{2 n}}^{*}$ 的 $q^{n}+1$ 阶子群. 令 $\zeta_{i} \in F_{i}^{(2)} \cap C$ 且 $\zeta_{0}=1$, 记 $\gamma_{i}:=\prod_{l=0}^{i} \zeta_{l}$. 令

$$
W:=\sum_{i=0}^{m-1} K_{i} \gamma_{i} \oplus F_{m} \cdot \gamma_{m}
$$

于是 $\left\{\rho_{\theta}(W): \theta \in C\right\}$ 是 $V=\mathbb{F}_{q^{2 n}}$ 中的一个辛展形, 并且该展形的全自同构群包含传递子群 $H=\left\langle\rho_{\theta}\right.$ : $\theta \in C\rangle$, 详情参见文献 [14].

\section{3 新的 $2^{9}$ 阶仿射平面的构造}

本节给出新的旗传递仿射平面的构造. 当素数 $p$ 及整数 $n$ 和 $f$ 满足条件 (2.2) 时, 已有结果证明 不可能出现任何让人意外的旗传递仿射平面. 为此, 表 1 列出 “较小” 的 $(p, n f)$ 对使得它们不满足条 件 (2.2). 这意味着在表 1 的情形下有可能出现不同于 $\mathcal{C}$ - 平面和 $\mathcal{H}$ - 平面的旗传递仿射平面. 此处不 列出 $(p, n f)=(3,5)$ 的情形是因为所有 125 阶以下的仿射平面已经被完全分类 (参见文献 [21]).

在接下来的讨论中, 考虑 $p=2$ 和 $n f=9$ 的情形. 令 $\gamma$ 是 $\mathbb{F}_{2^{18}}$ 的一个本原元使得它在 $\mathbb{F}_{2}$ 上的 极小多项式是 $X^{18}+X^{12}+X^{10}+X+1$. 令 $W$ 是 $\mathbb{F}_{2^{18}}$ 中具有下面形式的 3 维 $\mathbb{F}_{2^{3}}$ 线性子空间:

$$
W=\left\{x+y \xi: x \in \mathbb{F}_{2^{9}}, y \in \mathbb{F}_{2^{3}}, x+x^{2^{3}}+x^{2^{6}}=0\right\}, \quad \text { 其中 } \xi \in \mathbb{F}_{2^{18}} \backslash \mathbb{F}_{2^{9}} .
$$

令 $K$ 是 $\mathbb{F}_{2^{9}}$ 的迹映射 $x \mapsto x+x^{8}+x^{64}$ 的核, 则 $W$ 可以改写成 $K$ 和 $\mathbb{F}_{2^{3}} \cdot \xi$ 的直和, 即 $W=K \oplus \mathbb{F}_{2^{3}} \cdot \xi$.

引理 3.1 假定 $W$ 是如 (3.1) 中定义的线性空间, 则存在 $u, v \in \mathbb{F}_{2^{18}}$ 使得

$$
W=\left\{y \in \mathbb{F}_{2^{18}}: \operatorname{Tr}_{\mathbb{F}_{2^{18}} / \mathbb{F}_{2^{3}}}(u y)+\operatorname{Tr}_{\mathbb{F}_{2^{18}} / \mathbb{F}_{29}}(v y)=0\right\},
$$

其中 $\operatorname{Tr}_{\mathbb{F}_{2^{18}} / \mathbb{F}_{2^{3}}}$ 和 $\operatorname{Tr}_{\mathbb{F}_{2^{18}} / \mathbb{F}_{2^{9}}}$ 分别是从 $\mathbb{F}_{2^{18}}$ 到 $\mathbb{F}_{2^{3}}$ 和从 $\mathbb{F}_{2^{18}}$ 到 $\mathbb{F}_{2^{9}}$ 的迹线性变换.

表 1 一些不满足 $(2.2)$ 的 $(p, n f)$

\begin{tabular}{cccccc}
\hline$(p, n f)$ & $(2,9)$ & $(2,10)$ & $(3,10)$ & $(7,10)$ & $(11,3)$ \\
\hline $\operatorname{gcd}\left(p^{n f}+1,2 n f\right)$ & 9 & 5 & 5 & 10 & 6 \\
\hline
\end{tabular}


证明 考虑 $u, v \in \mathbb{F}_{2^{18}}$ 满足下面的方程组:

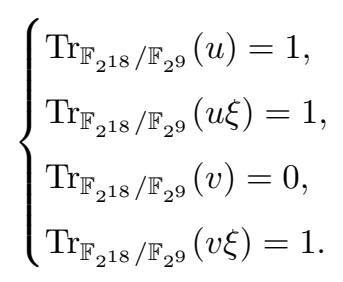

取 $\delta \in \mathbb{F}_{4}$ 使得 $\delta+\delta^{2}=1$. 因为 $\mathbb{F}_{2^{9}} \cap \mathbb{F}_{4}=\mathbb{F}_{2}$, 所以 $\{1, \delta\}$ 构成 $\mathbb{F}_{2^{18}}$ 的一组 $\mathbb{F}_{2^{9}}$ 线性基. 于是存 在 $a, b \in \mathbb{F}_{2^{9}}$ 使得 $\xi=a+b \delta$. 因此直接解方程组可得 $u=\frac{a+b+1}{b}+\delta, v=\frac{1}{b}$. 接着定义 $W_{1}=\{y \in$ $\left.\mathbb{F}_{2^{18}}: \operatorname{Tr}_{\mathbb{F}_{2^{18}} / \mathbb{F}_{2^{3}}}(u y)+\operatorname{Tr}_{\mathbb{F}_{2^{18}} / \mathbb{F}_{2^{9}}}(v y)=0\right\}$. 注意到 $W_{1}$ 中的每个元素 $y$ 都满足 $\operatorname{Tr}_{\mathbb{F}_{2^{18}} / \mathbb{F}_{29}}(v y) \in \mathbb{F}_{2^{3}}$, 即 $W_{1} \subseteq \mathbb{F}_{2^{9}} \oplus \mathbb{F}_{2^{3}} \cdot \xi$. 注意到 $1 \notin W_{1}$, 而且 $1 \in \mathbb{F}_{2^{9}}$, 我们有 $W_{1} \neq \mathbb{F}_{2^{9}} \oplus \mathbb{F}_{2^{3}} \cdot \xi$. 因为 $W_{1}$ 是 $\mathbb{F}_{2^{3}}$ 上的线 性空间, 所以 $W_{1}$ 在 $\mathbb{F}_{2^{3}}$ 上的维数小于等于 3 . 取 $\eta \in \mathbb{F}_{2^{9}}$ 满足 $\eta+\eta^{8}+\eta^{64}=0$, 故 $W$ 有一组 $\mathbb{F}_{2^{3}}$ 基 $\left\{\xi, \eta, \eta^{8}\right\}$. 通过直接验证可得 $\xi, \eta, \eta^{8} \in W_{1}$, 则 $W \subseteq W_{1}$. 证毕.

引理 3.2 令 $W$ 是如 (3.1) 中所示的线性空间. 如果对整数 $i$ 和 $j$ 有 $g=\rho^{i} \sigma^{j}$ 使得 $g(W)=W$, 那么 $i$ 能被 $\frac{2^{18}-1}{2^{3}-1}$ 整除.

证明 根据 (3.1) 下面的描述, 我们有 $W=K \oplus \mathbb{F}_{2^{3}} \cdot \xi$, 其中 $K=\left\{x \in \mathbb{F}_{2^{9}}: x+x^{8}+x^{64}=0\right\}$. 由引理 3.1 的表达式可知, $\operatorname{Tr}_{\mathbb{F}_{2^{18}} / \mathbb{F}_{2^{9}}}(v \cdot W) \subseteq \mathbb{F}_{2^{3}}$, 其中 $v \in \mathbb{F}_{2^{9}}$. 因为 $\sigma(K)=K$, 所以有

$$
\operatorname{Tr}_{\mathbb{F}_{2^{18} / \mathbb{F}_{2} 9}}(v \cdot g(W))=v K\left(\gamma^{i}+\gamma^{i \cdot 2^{9}}\right) \oplus v \mathbb{F}_{2^{3}} \cdot g\left(\xi+\xi^{2^{9}}\right) \subseteq \mathbb{F}_{2^{3}} .
$$

因此 $v K\left(\gamma^{i}+\gamma^{i \cdot 2^{9}}\right) \subseteq \mathbb{F}_{2^{3}}$. 比较以上两边的 $\mathbb{F}_{2^{3}}$ 维数可得 $\gamma^{i} \in \mathbb{F}_{2^{9}}^{*}$. 根据 $W \cap \mathbb{F}_{2^{9}}=K$ 可得 $g(K)=\rho^{i}(K)=K$, 故 $\rho^{i} \in\left\langle\rho^{\left(2^{18}-1\right) /\left(2^{3}-1\right)}\right\rangle$. 证毕.

现在开始构造 $V$ 中包含组件 $W$ 的展形 $\mathcal{S}$ 使得 $\Pi(\mathcal{S})$ 是一个可解旗传递仿射平面. 主要的想法是 假设 $\operatorname{Aut}(\mathcal{S})$ 有一个传递子群 $H$. 当选好这个传递子群 $H$ 后, 困难在于检查对任意 $g \in H \backslash \operatorname{Stab}_{W}(H)$ 有 $g(W) \cap W=\{0\}$. 我们用下面的方法来克服此困难. 根据引理 3.1 可知, 对任意 $g \in H \backslash \operatorname{Stab}_{W}(H)$ 有 $g(W) \cap W=\{0\}$ 当且仅当对任意 $y \in g(W)$ 有 $\operatorname{Tr}_{\mathbb{F}_{218} / \mathbb{F}_{2^{3}}}(u y)+\operatorname{Tr}_{\mathbb{F}_{2^{18}} / \mathbb{F}_{2^{9}}}(v y)=0$, 这意味着 $y=0$. 取 $W$ 的一组 $\mathbb{F}_{2^{3}}$ 基 $\left\{e_{1}, e_{2}, e_{3}\right\}$, 并记

$$
f_{i}:=\operatorname{Tr}_{\mathbb{F}_{2^{18}} / \mathbb{F}_{2^{3}}}\left(u g\left(e_{i}\right)\right)+\operatorname{Tr}_{\mathbb{F}_{2^{18}} / \mathbb{F}_{2^{9}}}\left(v g\left(e_{i}\right)\right), \quad \text { 其中 } i=1,2,3 .
$$

此时我们断定 $f_{1} 、 f_{2}$ 和 $f_{3}$ 是 $\mathbb{F}_{2^{9}}$ 的一组 $\mathbb{F}_{2^{3}-}$ 基, 否则存在 $a, b, c \in \mathbb{F}_{2^{3}}$ 使得 $a f_{1}+b f_{2}+c f_{3}=0$ 和 $a e_{1}+b e_{2}+c e_{3} \neq 0$. 故由文献 $[22$, 推论 2.38] 可得 $g(W) \cap W=\{0\}$ 当且仅当

$$
\left|\begin{array}{ccc}
f_{1} & f_{2} & f_{3} \\
f_{1}^{q} & f_{2}^{q} & f_{3}^{q} \\
f_{1}^{q^{2}} & f_{2}^{q^{2}} & f_{3}^{q^{2}}
\end{array}\right| \neq 0 \text {, 其中 } q=2^{3} \text {. }
$$

因为 $W=K \oplus \mathbb{F}_{2^{3}} \cdot \xi$, 所以可以取 $\left\{e_{1}, e_{2}, e_{3}\right\}=\left\{\eta, \eta^{8}, \xi\right\}$, 其中 $0 \neq \eta \in K$. 通过上述方法可以提高 检查 $g(W) \cap W$ 是否为 $\{0\}$ 的效率, 从而找到下面参数为 $(p, n, f, q)=(2,3,3,8)$ 的新例子.

定理 3.1 令 $\xi=\gamma^{9}$ 和 $W$ 如 (3.1) 中所示, 其中 $\gamma$ 在 $\mathbb{F}_{2}$ 上的极小多项式是 $X^{18}+X^{12}+X^{10}+X$ +1 . 定义 $a=6\left(2^{9}-1\right) /\left(2^{3}-1\right)+32$, 再令 $H=\left\langle\rho^{9\left(2^{9}-1\right) /\left(2^{3}-1\right)}, \rho^{a} \sigma^{2}\right\rangle$, 其中 $\rho^{a}$ 和 $\sigma$ 如 (2.1) 定义所 示. 那么 $\mathcal{S}=\{g(W): g \in H\}$ 是 $V$ 中的展形, 其中 $V=\mathbb{F}_{2^{18}}$ 被看作一个 6 维 $\mathbb{F}_{2^{3}}$ 线性空间. 
证明 利用 $\sigma \rho \sigma^{-1}=\rho^{2}$, 有 $\left(\rho^{a} \sigma^{2}\right)^{9}=\rho^{a\left(2^{18}-1\right) /\left(2^{2}-1\right)} \in\left\langle\rho^{9\left(2^{9}-1\right) /\left(2^{3}-1\right)}\right\rangle$. 因为 $\left\langle\rho^{9\left(2^{9}-1\right) /\left(2^{3}-1\right)}\right\rangle$ 是 $H$ 的正规子群, 所以 $|H|=\left(2^{3}-1\right)\left(2^{9}+1\right)$. 取 $g=\rho^{i} \sigma^{j} \in H$ 使得 $g(W)=W$. 由引理 3.2 可 知, $\rho^{i} \in\left\langle\rho^{\left(2^{18}-1\right) /\left(2^{3}-1\right)}\right\rangle$. 注意到 $W$ 是 $\mathbb{F}_{2^{3}}$ 线性的, 我们有 $g(W)=\sigma^{j}(W)=K \oplus \mathbb{F}_{2^{3}} \cdot \sigma^{j}\left(\gamma^{9}\right)$. 借助 Magma ${ }^{[23]}$, 对于 $0 \leqslant j \leqslant 17$, 有 $\sigma^{j}\left(\gamma^{9}\right) \in W$ 当且仅当 $j=0$. 因此 $\operatorname{Stab}_{W}(H)=\left\langle\rho^{\left(2^{18}-1\right) /\left(2^{3}-1\right)}\right\rangle$, 于是 $|\mathcal{S}|=2^{9}+1$. 剩下只需要验证对任意 $g \in H$ 且 $g \notin\left\langle\rho^{\left(2^{18}-1\right) /\left(2^{3}-1\right)}\right\rangle$ 都有 $(3.2)$ 成立, 具体的验证通过 Magma ${ }^{[23]}$ 实现.

推论 3.1 令 $\mathcal{S}$ 是来自定理 3.1 的展形, 则 $\operatorname{ker}(\mathcal{S})=\mathbb{F}_{2^{3}}$.

证明 根据 $\operatorname{ker}(\mathcal{S})$ 的定义可知, $\mathcal{S}$ 的每个组件都是 $\operatorname{ker}(\mathcal{S})$ - 模. 由引理 3.1 可知, 对于每个 $W \in \mathcal{S}$, $W$ 是 $\mathbb{F}_{2^{3}}$ 线性的, 并且不可能在 $\mathbb{F}_{2^{18}}$ 中更大的子域上是线性的, 因此 $\operatorname{ker}(\mathcal{S})=\mathbb{F}_{2^{3}}$.

定理 3.2 令 $\mathcal{S}$ 和 $H$ 是在定理 3.1 中给出的展形和它的一个传递子群, 则展形 $\mathcal{S}$ 的全自同构群 即为 $H$, 其中 $H$ 包含一个正规子群 $H_{0}=\left\langle\rho^{9\left(2^{9}-1\right) /\left(2^{3}-1\right)}\right\rangle$ 使得 $H / H_{0} \cong Z_{9}$.

证明 由 Foulser ${ }^{[7]}$ 的结果可知, 如果旗传递仿射平面的自同构群可解, 那么对应的展形的自同 构群同构于 $\Gamma \mathrm{L}\left(1, q^{2 n}\right)$ 的子群. 因为 $H \leqslant \operatorname{Aut}(\mathcal{S}) \leqslant\langle\rho, \sigma\rangle$, 并且 $H$ 在 $\mathcal{S}$ 的组件上是传递的, 所以有

$$
\frac{|H|}{\left|\operatorname{Stab}_{W}(H)\right|}=\frac{|\operatorname{Aut}(\mathcal{S})|}{\left|\operatorname{Stab}_{W}(\operatorname{Aut}(\mathcal{S}))\right|}=2^{9}+1
$$

由定理 3.1 的证明可得

$$
\operatorname{Stab}_{W}(\langle\rho, \sigma\rangle)=\left\langle\rho^{\left(2^{18}-1\right) /\left(2^{3}-1\right)}\right\rangle,
$$

于是 $\operatorname{Stab}_{W}(H)=\operatorname{Stab}_{W}(\operatorname{Aut}(\mathcal{S}))$. 因此 $H=\operatorname{Aut}(\mathcal{S})$. 最后根据定理 3.1 的证明的第一部分可知 $H_{0}:=H \cap\langle\rho\rangle=\left\langle\rho^{9\left(2^{9}-1\right) /\left(2^{3}-1\right)}\right\rangle$ 是 $H$ 的正规子群, 并且 $\left(\rho^{a} \sigma^{2}\right)^{9} \in H_{0}$, 因此有 $H / H_{0} \cong Z_{9}$.

注 3.1 令 $\mathcal{S}$ 是定理 3.1 中给出的展形. 根据定理 3.2 可知 $\operatorname{Aut}(\mathcal{S}) \cap\langle\rho\rangle=\left\langle\rho^{9\left(2^{9}-1\right) /\left(2^{3}-1\right)}\right\rangle, \Pi(\mathcal{S})$ 不是 $\mathcal{C}$ - 平面, 因此不可能来自于 Kantor-Williams 构造 ${ }^{[14]}$ 。除此之外, 我们也找到两个来自 KantorWilliam 构造的 $\mathcal{C}$ - 平面, 但是没有找到包含如 (3.1) 所示的组件 $W$ 使得 $\operatorname{Aut}\left(\mathcal{S}^{\prime}\right) \cap\langle\rho\rangle=\left\langle\rho^{3\left(2^{9}-1\right) /\left(2^{3}-1\right)}\right\rangle$ 的展形 $\mathcal{S}^{\prime}$.

\section{4 总结}

借助深刻的置换群理论, 旗传递射影平面的分类工作已经取得了足够大的进展, 参见文献 [24,25]. 相比之下, 旗传递仿射平面具有更多的可能性, 完整的分类以目前的技术仍然是遥不可及的. 本文首次 给出了不同构于 $\mathcal{C}$ - 平面或 $\mathcal{H}$ - 平面的可解旗传递仿射平面. 这意味着, 在条件 $(2.2)$ 不满足时, 旗传递仿 射平面的确有更多的可能性. 特别地, 考虑 $q^{n}=2^{9}$ 和 $n f=9$ 的情形, 我们有 $\operatorname{gcd}\left(q^{n}+1,2 n f\right)=9>1$, 这暗示除了 $\mathcal{C}$ - 平面以外还有另外的可能: Singer 子群 $\operatorname{Aut}(\mathcal{S}) \cap\langle\rho\rangle$ 的轨道是大小为 $\frac{\left(q^{n}+1\right)}{3}$ 或 $\frac{\left(q^{n}+1\right)}{9}$ 的部分展形. 但是计算搜索结果显示, 当具有如 (3.1) 的组件的展形的自同构群包含 $\left\langle\rho^{3\left(2^{9}-1\right) /\left(2^{3}-1\right)}\right\rangle$ 时, 由该展形得到的平面是 $\mathcal{C}$ - 平面 (见注 3.1), 即这种情形不存在 “吕” 的部分展形. 因为我们的计算 机搜索并不完全, 所以不能排除这类平面存在的可能性, 于是进一步完整地给出 $2^{9}$ 阶旗传递仿射平面 的分类是相当有意义的, 这对解决可解旗传递仿射平面的分类问题有重要的帮助. 同时我们也注意到 $\operatorname{gcd}\left(2^{3^{t}}+1,2 \cdot 3^{t+1}\right)=3^{t+1}$, 能否借助 (3.1) 的检验方法将定理 3.1 的例子推广成一类 $2^{3^{t}}$ 阶旗传递仿 射平面也是一个十分有意思的问题.

致谢 感谢审稿人提出的宝贵意见. 


\section{参考文献}

1 Lüneburg H. Translation Planes. Berlin-New York: Springer-Verlag, 1980

2 Maschietti A. Symplectic translation planes. Lect Notes Semin Interdiscip Mat, 2003, 2: 101-148

3 André J. Über nicht-Desarguessche Ebenen mit transitiver Translationsgruppe. Math Z, 1954, 60: 156-186

4 Wagner A. On finite affine line transitive planes. Math Z, 1965, 87: 1-11

5 Buekenhout F, Delandtsheer A, Doyen J, et al. Linear spaces with flag-transitive automophism groups. Geom Dedicata, 1990, 36: 89-94

6 Liebeck M W. The classification of finite linear spaces with flag-transitive automorphism groups of affine type. J Combin Theory Ser A, 1998, 84: 196-235

7 Foulser D A. Solvable flag transitive affine groups. Math Z, 1964, 86: 191-204

8 Foulser D A. The flag-transitive collineation groups of the finite Desarguesian affine planes. Canad J Math, 1964, 16: 443-472

9 Kantor W M. Two families of flag-transitive affine planes. Geom Dedicata, 1992, 41: 191-200

10 Kantor W M, Suetake C. A note on some flag-transitive affine planes. J Combin Theory Ser A, 1994, 65: 307-310

11 Suetake C. Flag transitive planes of order $q^{n}$ with a long cycle $l_{\infty}$ as a collineation. Graphs Combin, 1991, 7: 183-195

12 Suetake C. On flag-transitive affine planes of order $q^{3}$. Geom Dedicata, 1994, 51: 123-131

13 Feng T. Finite flag-transitive affine planes with a solvable automorphism group. J Combin Theory Ser A, 2017, 152: $225-254$

14 Kantor W M, Williams M E. New flag-transitive affine planes of even order. J Combin Theory Ser A, 1996, 74: 1-13

15 Baker R D, Ebert G L. Construction of two-dimensional flag-transitive planes. Geom Dedicata, 1988, 27: 9-14

16 Baker R D, Ebert G L. Two-dimensional flag-transitive planes revisited. Geom Dedicata, 1996, 63: 1-15

17 Baker R D, Dover J M, Ebert G L, et al. Perfect Baer subplane partitions and three-dimensional flag-transitive planes. Des Codes Cryptogr, 2000, 21: 19-39

18 Baker R D, Culbert C, Ebert G L, et al. Odd order flag-transitive affine planes of dimension three over their kernel. Adv Geom, 2003, 3: 215-223

19 Baker R D, Ebert G L, Leung K H, et al. A trace conjecture and flag-transitive affine planes. J Combin Theory Ser A, 2001, 95: 158-168

20 Ebert G L. Partitioning problems and flag-transitive planes. Rend Circ Mat Palermo (2) Suppl, 1998, 53: 27-44

21 Prince A R. Flag-transitive affine planes of order at most 125. J Geom, 2000, 67: 208-216

22 Lidl R, Niederreiter H. Finite Fields. Encyclopedia of Mathematics and Its Applications, vol. 20. Cambridge: Cambridge University Press, 1997

23 Bosma W, Cannon J, Fieker C, et al. Handbook of Magma Functions. Http://magma.maths.usyd.edu.au/magma/ handbook/, 2013

24 Kantor W M. Primitive permutation groups of odd degree, and an application to finite projective planes. J Algebra, 1987, 106: 15-45

25 Thas K, Zagier D. Finite projective planes, Fermat curve, and Gaussian period. J Eur Math Soc (JEMS), 2008, 10: $173-190$

\section{附录 A Magma 代码}

下面提供验证定理 3.1 的 Magma 代码, 而对于更一般的情形只需要遍历 $\xi \in \mathbb{F}_{2^{18}} \backslash \mathbb{F}_{2^{9}}$ 和 $0 \leqslant a$ $<d \frac{2^{9}-1}{2^{3}-1}$, 其中 $d \in\{1,3,9\}$.

$\mathrm{F}:=\mathrm{GF}(512) ; \mathrm{E}<g a>:=\operatorname{ext}<\mathrm{F} \mid 2>$;

$\mathrm{R}<X>$ :=PolynomialRing $(\mathrm{GF}(2))$;

MinimalPolynomial(ga) eq $X^{18}+X^{12}+X^{10}+X+1$;

delta: $=\mathrm{GF}(4) .1 ;$ eta: $=$ Trace $(\mathrm{F} \cdot 1, \mathrm{GF}(8))+\mathrm{F} .1$;

$\mathrm{w}:=\mathrm{ga}^{\wedge}\left(\left(2^{9}-1\right) \operatorname{div}\left(2^{3}-1\right)\right) ; \mathrm{xi}:=\mathrm{ga} \wedge$;

$\mathrm{xi0}:=\mathrm{xi}^{*} \operatorname{delta}^{2}+\mathrm{xi}^{\wedge}\left(2^{9}\right)^{*} \operatorname{delta} ; \mathrm{xi1}:=\mathrm{xi}+\mathrm{xi}^{\wedge}\left(2^{9}\right)$;

$\mathrm{u}:=(\mathrm{xi0}+\mathrm{xi1}+1) / \mathrm{xi1}+\operatorname{delta} ; \mathrm{v}:=\mathrm{xi1}{ }^{\wedge}(-1) ; \mathrm{a}:=6^{*}\left(2^{9}-1\right) /\left(2^{3}-1\right)+32$;

$\mathrm{W}:=\left\{\mathrm{x}+\mathrm{xi}{ }^{*} \mathrm{y}: \mathrm{x}\right.$ in $\mathrm{F}, \mathrm{y}$ in $\mathrm{GF}(8) \mid \operatorname{Trace}(\mathrm{x}, \mathrm{GF}(8))$ eq 0$\}$; 
$/ /$ 验证 $\sigma^{j}\left(\gamma^{9}\right) \in W$ 当且仅当 $j=0$, 其中 $0 \leqslant j \leqslant 17$.

$\left\{\mathrm{xi}^{\wedge}\left(2^{i}\right)\right.$ : i in $\left.[0 . .17]\right\}$ meet $\mathrm{W}$;

$/ / \mathrm{H}$ 中群元素可以表示成 $\rho^{i}\left(\rho^{a} \sigma^{j}\right)$, 即 $[\mathrm{i}, \mathrm{j}]$ 对

$\mathrm{H}:=\left\{[\mathrm{i}, \mathrm{j}]\right.$ : i in $\left[1 . .\left(2^{3}-1\right) *\left(2^{9}+1\right) \operatorname{div}\left(2^{3}+1\right)\right], \mathrm{j}$ in $\left.[0 . .8]\right\}$;

//定义函数若 (3.2) 成立返回 true, 否则返回 false

IsDisjoint: $=$ function $(\mathrm{u}, \mathrm{v}, \mathrm{xi}, \mathrm{h})$

$\mathrm{e} 1:=\mathrm{w}^{\wedge}\left(9^{*} \mathrm{~h}[1]\right) * \mathrm{ga}^{\wedge}\left(\mathrm{a}^{*}\left(4^{\wedge} \mathrm{h}[2]-1\right) \operatorname{div}(4-1)\right)^{*} \mathrm{xi}^{\wedge}\left(4^{\wedge}(\mathrm{h}[2])\right)$;

$\mathrm{e} 2:=\mathrm{w}^{\wedge}\left(9^{*} \mathrm{~h}[1]\right) * \mathrm{ga}^{\wedge}\left(\mathrm{a}^{*}\left(4^{\wedge} \mathrm{h}[2]-1\right) \operatorname{div}(4-1)\right)^{*} \operatorname{eta}^{\wedge}\left(4^{\wedge}(\mathrm{h}[2])\right)$

$\mathrm{e} 3:=\mathrm{w}^{\wedge}\left(9^{*} \mathrm{~h}[1]\right)^{*} \mathrm{ga}^{\wedge}\left(\mathrm{a}^{*}\left(4^{\wedge} \mathrm{h}[2]-1\right) \operatorname{div}(4-1)\right)^{*} \operatorname{eta}^{\wedge}\left(8^{*} 4^{\wedge}(\mathrm{h}[2])\right)$;

$\mathrm{f} 1:=\operatorname{Trace}\left(\mathrm{u}^{*} \mathrm{e} 1, \operatorname{GF}(8)\right)+\operatorname{Trace}\left(\mathrm{v}^{*} \mathrm{e} 1, \mathrm{GF}(512)\right)$;

$\mathfrak{f} 2:=\operatorname{Trace}\left(\mathrm{u}^{*} \mathrm{e} 2, \mathrm{GF}(8)\right)+\operatorname{Trace}\left(\mathrm{v}^{*} \mathrm{e} 2, \mathrm{GF}(512)\right) ;$

f3:=Trace $\left(\mathrm{u}^{*} \mathrm{e} 3, \mathrm{GF}(8)\right)+\operatorname{Trace}\left(\mathrm{v}^{*} \mathrm{e} 3, \mathrm{GF}(512)\right)$;

$\mathrm{M}:=\operatorname{Matrix}\left(\mathrm{F}, 3,3,\left[\mathrm{f1}, \mathrm{f} 2, \mathrm{f3}, \mathrm{f1} 1^{\wedge} 8, \mathrm{f2} 2^{\wedge} 8, \mathrm{f3}{ }^{\wedge} 8, \mathrm{f1} 1^{\wedge} 64, \mathrm{f2} 2^{\wedge} 64, \mathrm{f3} 3^{\wedge} 64\right]\right)$;

if Determinant(M) ne 0 then return true; else return false; end if;

end function;

$\mathrm{H} 1:=\{\mathrm{h}: \mathrm{h}$ in $\mathrm{H} \mid$ IsDisjoint $(\mathrm{u}, \mathrm{v}, \mathrm{xi}, \mathrm{h})$ eq false $\}$;

$/ /$ 验证对任意 $g \in H$ 且 $g \notin\left\langle\rho^{\left(2^{18}-1\right) /\left(2^{3}-1\right)}\right\rangle$ 都有 (3.2) 成立

H1 eq $\{[(512+1) \operatorname{div} 9 * i, 0]$ : i in $[1 . .7]\}$;

\section{A new flag-transitive affine plane of order $2^{9}$}

\section{Tao Feng, Weicong Li \& Qing Xiang}

Abstract Finite flag-transitive affine planes have received much attention during the past fifty years because of their connections with other combinatorial objects such as spreads, planar functions, semifields and linearized polynomials. In 1964, Foulser completely determined the automorphism groups of finite flag-transitive affine planes. If a flag-transitive affine plane has a solvable automorphism group, then the affine plane is called solvable. The non-solvable flag-transitive affine planes have been completely classified in the 1990s. But the complete classification for the solvable case seems far out of reach. All known solvable flag-transitive affine planes can be classified into two types: $\mathcal{C}$-planes and $\mathcal{H}$-planes, where $\mathcal{H}$-planes only occur in the odd characteristic case. In this paper, we construct the first flag-transitive affine plane of order $2^{9}$ over its kernel $\mathbb{F}_{2^{3}}$, which is not of type $\mathcal{C}$ and the largest Singer subgroup of the translation complement has order $\left(2^{3}-1\right)\left(2^{9}+1\right) / 9$.

Keywords affine plane, flag-transitive, spread, translation plane

MSC(2020) 51E15, 05B25, 51A40

doi: 10.1360/SSM-2021-0033 